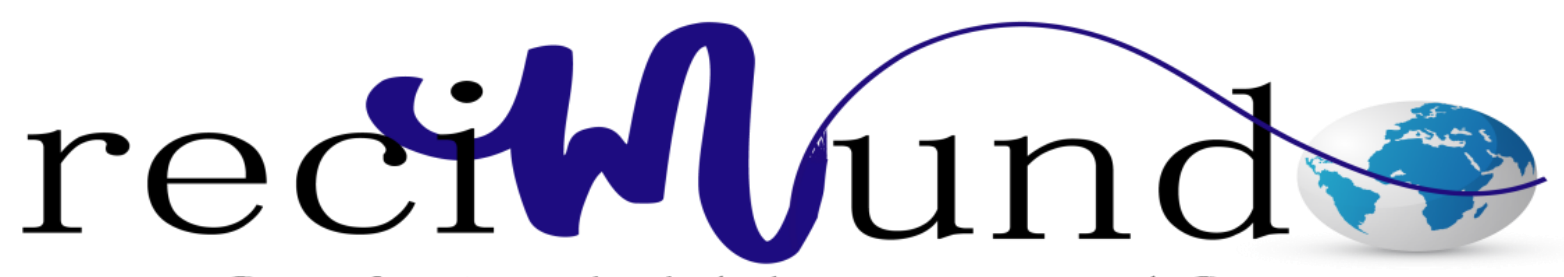

Revista Científica Mundo de la Investigación y el Conocimiento

Yessenia Isabel Sarchi Guayasamin a; Olmedo Xavier Ruiz Lara ${ }^{\text {b; }}$ Paulina

Elizabeth Durán Mora ${ }^{c}$; Jessica Karina Herrera Jaramillo ${ }^{\text {d }}$

Limpiezas quirúrgicas en pacientes quemados

Surgical cleanings in burned patients

Revista Científica Mundo de la Investigación y el Conocimiento. Vol. 3 núm.3, septiembre, ISSN: 2588-073X, 2019, pp. 1303-1326

DOI: 10.26820/recimundo/3.(3).septiembre.2019.1303-1326

URL: http://recimundo.com/index.php/es/article/view/571

Código UNESCO: 3205 Medicina Interna

Tipo de Investigación: Artículo de Revisión

Editorial Saberes del Conocimiento

Recibido: $15 / 05 / 2019$

Aceptado: 23/06/2019

Publicado: 30/09/2019

Correspondencia: yesseniasarchi30familia@gmail.com

a. Médico General; Hospital Marco Vinicio Iza; Servicio Medicina Interna Médico Residente; Lago Agrio, Ecuador; yesseniasarchi30familia@gmail.com

b. Médico General; Servicio de Traumatología; Hospital General Ibarra; Ibarra; olxarula@ gmail.com

c. Médico General; Servicio Cirugía General; Hospital General Privado Durán; Ambato, Ecuador; paupau_edm@hotmail.com

d. Médico General en Funciones Hospitalarias; Servicio de Medicina Interna; Hospital General Marco Vinicio Iza; Lago Agrio, Ecuador; jessiherrera1989@ hotmail.com 


\section{Limpiezas quirúrgicas en pacientes quemados}

Vol. 3, núm. 3., (2019)

Yessenia Isabel Sarchi Guayasamin; Olmedo Xavier Ruiz Lara; Paulina Elizabeth Durán Mora; Jessica Karina Herrera Jaramillo

\section{RESUMEN}

El principal procedimiento en la atención de una quemadura está constituido por la limpieza, que da pie al diagnóstico y tratamiento de la lesión, por tal razón resulta fundamental realizarlo adecuadamente, dado que es la piedra angular del manejo del paciente quemado. Las quemaduras representan un problema de salud importante en todo el mundo, por tanto, merece especial atención todo lo concerniente a este tema. Comúnmente se deben a las escaldaduras por líquidos calientes y vapor, los incendios en edificios y los líquidos y gases inflamables, entre otras. Son los casos de quemadas de mayor gravedad las que requerirán una limpieza más profunda, llevada a cabo por medio de cortes o láser, llamada limpieza quirúrgica, definida como la que abarca la limpieza de la piel con antisépticos, la irrigación de las heridas con solución salina y el desbridamiento quirúrgico del tejido muerto y todos los cuerpos extraños. El objetivo de la presente investigación documental, se centra en efectuar una revisión a la literatura científico académica reciente, mediante búsqueda de fuentes bibliográficas en las referidas bases de datos, con la finalidad de explicar de qué se trata la limpieza quirúrgica en pacientes quemados. Los resultados estriban en la exposición de algunos de los conceptos elementales relativos a la afección propiamente sufrida por el paciente, y además se logra explicar de manera concisa lo que se entiende por limpieza quirúrgica. En conclusión, se confirma que la limpieza quirúrgica permite, fundamentalmente, preparar el área para realizar injertos, sobretodo en casos de quemaduras de mayor gravedad, aumentado así las posibilidades de supervivencia, entre otros beneficios, por lo tanto, puede afirmarse que este procedimiento representa un papel fundamental en el manejo de las quemaduras, y es uno de los más importantes en la medicina actual.

Palabras Claves: Lesión; Irrigación; Desbridamiento; Tejido; Supervivencia. 


\title{
Limpiezas quirúrgicas en pacientes quemados
}

Vol. 3, núm. 3., (2019)

Yessenia Isabel Sarchi Guayasamin; Olmedo Xavier Ruiz Lara; Paulina Elizabeth Durán Mora;

Jessica Karina Herrera Jaramillo

\begin{abstract}
The main procedure in the care of a burn is constituted by cleaning, which gives rise to the diagnosis and treatment of the injury, for this reason it is essential to perform it properly, since it is the cornerstone of the management of the burned patient. Burns represent a major health problem worldwide, therefore, everything concerning this issue deserves special attention. They are commonly due to scalds due to hot liquids and steam, building fires and flammable liquids and gases, among others. It is the cases of burns of greater severity that will require a deeper cleaning, carried out by means of cuts or laser, called surgical cleaning, defined as the one that covers the cleaning of the skin with antiseptics, the irrigation of wounds with solution saline and surgical debridement of dead tissue and all foreign bodies. The objective of the present documentary investigation is focused on carrying out a review of the recent scientific and academic literature, through the search of bibliographic sources in the aforementioned databases, in order to explain what surgical cleaning is about in burn patients. The results are based on the presentation of some of the elementary concepts related to the condition itself suffered by the patient, and it is also possible to explain in a concise way what is understood by surgical cleaning. In conclusion, it is confirmed that surgical cleaning allows, fundamentally, to prepare the area for grafting, especially in cases of burns of greater severity, thus increasing the chances of survival, among other benefits, therefore, it can be stated that this procedure represents a fundamental role in the management of burns, and is one of the most important in current medicine.
\end{abstract}

Key words: Injury; Irrigation; Debridement; Tissue; Survival. 


\section{Limpiezas quirúrgicas en pacientes quemados}

Vol. 3, núm. 3., (2019)

Yessenia Isabel Sarchi Guayasamin; Olmedo Xavier Ruiz Lara; Paulina Elizabeth Durán Mora; Jessica Karina Herrera Jaramillo

\section{Introducción.}

Las quemaduras representan un problema de salud importante en todo el mundo, por tanto, merece especial atención todo lo concerniente a este tema. Se define como quemadura aquella lesión en los tejidos del cuerpo originadas por las siguientes causas: calor, electricidad, sustancias químicas, sol o radiación. Se tienen como las causas más comunes: "las escaldaduras por líquidos calientes y vapor, los incendios en edificios y los líquidos y gases inflamables. Otro tipo de quemadura es la causada por inhalación de humo o partículas tóxicas”. (Enciclopedia Médica ADAM, 2019)

Es necesario resaltar que un aproximado de 180.000 muertes al año se producen en todo el mundo a causa de las quemaduras. Estas en su mayoría tienen lugar en países de ingreso bajo y mediano. (Organización Mundial de la Salud, 2018)

En este orden de ideas, cabe destacar que un buen diagnóstico constituye la base fundamental de un buen tratamiento y, por ende, de la mejor recuperación del paciente quemado. Para ello es fundamental clasificar las quemaduras, que en base a su mecanismo de producción pueden ser: solares, por la radiación electromagnética que procede del sol; por contacto accidental con líquidos a temperaturas elevadas, siendo una de las causas más frecuentes de quemaduras graves debido a que los líquidos calientes se expanden rápidamente por la superficie cutánea, permitiendo que el mismo penetre más fácilmente hasta las capas más profundas, y de éstos destacándose aquellos líquidos de naturaleza grasa, por poseer una mayor adherencia a la piel, y por lo tanto, concibiéndose como uno de los más dañinos; por vapores y gases, generalmente producidos por la combustión o ebullición de diversas sustancias; químicas, causadas por contacto 


\section{Limpiezas quirúrgicas en pacientes quemados}

Vol. 3, núm. 3., (2019)

Yessenia Isabel Sarchi Guayasamin; Olmedo Xavier Ruiz Lara; Paulina Elizabeth Durán Mora; Jessica Karina Herrera Jaramillo

con sustancias cáusticas, ácidas o alcalinas; por electricidad, que a su vez se subdivide en otros dos tipos: por contacto, en el que una pequeña pero profunda zona cutánea se afecta, con una característica destrucción de tejidos que, en fin, se separarán y desprenderán; y por fogonazo, que afectan superficialmente a una zona más extensa de la piel, y por último están las producidas por fuego directo. (González, 2003)

Así mismo, pueden clasificarse por su grado de profundidad en: quemaduras de primer grado, que representa la de menor gravedad, por cuanto afecta solamente la capa externa de la piel. Las quemaduras de segundo grado, que son más graves que las anteriores, por cuanto afectan la capa externa y la capa media de la piel [algunos autores, subdividen este grado de quemadura en dos más, que son: las superficiales y las profundas]. Por último, las quemaduras de tercer grado, siendo éstas las de mayor gravedad ya que afectan las capas externa, media e interna de la piel. (Enciclopedia Médica ADAM, 2019). Luego de este grado, igualmente otros autores se refieren a un cuarto grado de quemaduras, relativas a la destrucción de músculos y estructuras óseas.

El principal procedimiento en la atención de una quemadura está constituido por la limpieza, que da pie al diagnóstico y tratamiento de la lesión, por tal razón resulta fundamental realizarlo adecuadamente dado que es la piedra angular del manejo del paciente quemado.

En aquellos casos de quemadas de mayor gravedad (segundo y tercer grado) requerirán una limpieza más profunda, llevada a cabo por medio de cortes o láser, llamada limpieza quirúrgica. La OMS (2007) define la limpieza quirúrgica como aquella que abarca la limpieza de la piel con antisépticos, la irrigación de las heridas con solución salina y el desbridamiento quirúrgico del tejido muerto y todos los cuerpos extraños. (p. 10) 


\section{Limpiezas quirúrgicas en pacientes quemados}

Vol. 3, núm. 3., (2019)

Yessenia Isabel Sarchi Guayasamin; Olmedo Xavier Ruiz Lara; Paulina Elizabeth Durán Mora; Jessica Karina Herrera Jaramillo

Asimismo, la Enciclopedia Médica ADAM (2018) define el desbridamiento como aquel "proceso de eliminación de piel y tejido muertos. Este tejido debe eliminarse para ayudar a que la herida sane. Hay muchas maneras de hacer esto. Puede necesitar anestesia general (dormido y sin dolor) para el desbridamiento de una herida grande".

La importancia de un buen manejo de las lesiones ocasionadas en quemados radica en el impacto tanto físico como psíquico, que se agrava por el recuerdo del trauma vivido por el paciente, aunado al sufrimiento y dolor del cuidado que revisten estas lesiones y su tratamiento. El estado de salud del paciente quemado también influye en la calidad de vida posterior, considerando que las secuelas alteran la imagen corporal, algunas veces su función normal, así como la autopercepción, e incluso su autoestima. (Servicio Andaluz de Salud de España, 2011)

Por tal razón, proporcionar los más adecuados cuidados a estos pacientes, sobre todo a aquellos de mayor gravedad, es fundamental para la prevención de complicaciones y la reducción del gasto clínico, así como para la mejor recuperación del paciente quemado.

El objetivo primordial de la presente investigación es plasmar lo relacionado con la limpieza quirúrgica en pacientes quemados, sin embargo, a continuación, se procede a desarrollar aspectos igualmente relevantes tales como: qué se entiende por quemadura, cuál es la sintomatología básica asociada, como se concibe el grado de daño por quemaduras, y finalmente, cuál es la metodología clínica relativa a la limpieza quirúrgica.

\section{Materiales y métodos}




\section{Limpiezas quirúrgicas en pacientes quemados}

Vol. 3, núm. 3., (2019)

Yessenia Isabel Sarchi Guayasamin; Olmedo Xavier Ruiz Lara; Paulina Elizabeth Durán Mora; Jessica Karina Herrera Jaramillo

Principalmente se realiza una búsqueda aleatoria y consecutiva de la literatura en diversas bases de datos, entre las que destacaron: EBSCO, PubMed, LILACS, Embase, SciELO, MedlinaPlus, usando expresiones tales como "Limpieza quirúrgica en pacientes quemados", "Limpieza quirúrgica en quemaduras", "Limpieza quirúrgica de quemados", "limpieza de quemados", entre otros términos; lo que aproximadamente resultó en menos de una decena de miles de registros bibliográficos. Luego, fue necesario que éstos resultados se redujesen, por ello se fueron aplicando filtros en base a criterios de: idioma español e inglés, relevancia, correlación temática y fecha de publicación en los últimos diez años (salvo algunas excepciones), rescatando todo aquel material que se refería a artículos científicos, guías clínicas, e-books, ensayos clínicos, consensos, protocolos, revisiones sistemáticas con o sin metaanálisis, estudios clínicos aleatorizados, estudios observacionales, boletines y/o folletos de reconocidas instituciones (públicas y privadas), tesis de posgrado y doctorado, noticias científicas y demás documentos e informaciones que, en base al propio criterio de este equipo, se asumió como de destacado interés y/o considerable valor científico por la categorización de la evidencia mostrada. Se desestimaron estudios de cohorte, casos y controles, series y reportes de casos, editoriales, cartas al editor y otros tipos de materiales bibliográficos que igualmente, a juicio de este equipo, se consideraron de escaso valor científico académico o nivel de evidencia percibido de en sus contenidos.

Es por lo tanto que, de lo antes dicho, puede llegar a afirmarse que el objetivo de la presente investigación se centra en efectuar una revisión a la literatura cientificoacadémica reciente disponible, mediante búsqueda de referencias bibliográficas en las referidas bases de datos, con la finalidad de explicar de qué se trata la limpieza quirúrgica en pacientes quemados. 


\section{Limpiezas quirúrgicas en pacientes quemados}

Vol. 3, núm. 3., (2019)

Yessenia Isabel Sarchi Guayasamin; Olmedo Xavier Ruiz Lara; Paulina Elizabeth Durán Mora; Jessica Karina Herrera Jaramillo

En el mismo orden de ideas, cabe mencionar que paralelamente se efectuó una búsqueda sin limitación cronológica ni de nivel de evidencia para identificar información que, considerando pudiera ser eventualmente entendida como un tanto distinta del objeto sobreentendido en el título de este tema, resulta es igualmente trascendente para comprender lo que aquí se quiere exponer, es decir, que se trata del contenido general y fundamental para el desarrollo de la presente revisión.

En definitiva, fue posterior al proceso antes descrito que el equipo investigador le da la correspondiente lectura crítica y análisis interpretativo a toda la evidencia determinada, resultando entonces todo este sumario, el fundamento de las ideas aquí expresadas de manera consensuada.

La inclusión o exclusión de cada una de las referencias en la presente revisión se definió igualmente por el consenso todos los participantes en este proceso investigativo, y de la misma manera fueron resueltas las decisiones disímiles.

\section{Resultados}

Para instituciones como Mayo Clinic (2019), las quemaduras, son entendidas como complicaciones médicas que significan una lesión sobre una o varias capas de tejido, que básicamente pueden categorizarse entre menor y grave, pudiendo éste último caso constituir una posible situación de emergencia potencialmente fatal. Estos daños se asocian al "calor, la sobreexposición al sol o a otra radiación, o el contacto con productos químicos o la electricidad".

Córdova, Moreno, Maruri, \& Criollo (2019) citando el trabajo de (Ambrosoni, 2018) definen la quemadura como "una lesión producida en la piel u otro tejido por agentes físicos, 


\section{Limpiezas quirúrgicas en pacientes quemados}

Vol. 3, núm. 3., (2019)

Yessenia Isabel Sarchi Guayasamin; Olmedo Xavier Ruiz Lara; Paulina Elizabeth Durán Mora; Jessica Karina Herrera Jaramillo

químicos o biológicos capaces de generar trastornos que van desde el simple eritema hasta la destrucción total o parcial de los tejidos afectados" (p. 111)

\section{Síntomas}

En base al grado del daño en piel es que pueden variar ésos síntomas. En los casos de graves quemaduras, el desarrollo de dichos síntomas, o signos, pueden llegar a tardar uno o dos días. (Mayo Clinic, 2019)

Estos pueden manifestarse como: ampollas que están intactas (sin romperse) o tienen rupturas que están perdiendo fluido, dolor (que, por cierto, no se encuentra relacionado con la gravedad de la quemadura, pues pueden las más graves ser indoloras), peladuras en la piel, estados de Shock o choque (caracterizado por la palidez, piel fría y húmeda, debilidad, labios y uñas azulados y disminución de la lucidez mental), inflamación, piel blanca, roja o carbonizada; y en los casos de quemado de las vías respiratorias, pueden presentarse: quemaduras en la cabeza, rostro, cuello, cejas o vellos nasales, boca y labios, tos, dificultad para respirar, moco oscuro (con manchas negras), cambios en la voz, sibilancias. (Medline Plus, 2018)

Tipos de quemaduras

En función de su causa, Culleiton \& Simko (2013) indican que una lesión por quemadura puede ser: térmica, química, eléctrica, radiación, humo o inhalación, o congelación, que explican de la siguiente manera. 


\section{Limpiezas quirúrgicas en pacientes quemados}

Vol. 3, núm. 3., (2019)

Yessenia Isabel Sarchi Guayasamin; Olmedo Xavier Ruiz Lara; Paulina Elizabeth Durán Mora; Jessica Karina Herrera Jaramillo

\section{Quemaduras térmicas}

Se originan por el contacto con sustancias calientes, incluyendo las ocasionadas por llamas, objetos sólidos calientes y vapor, que causan daño celular por coagulación. En este tipo de quemaduras, la herida será más profunda cuando mayor sea el tiempo de contacto de éstas sustancias con la piel. Particularmente, serán más profundas aquellas quemaduras que son por contacto con líquidos a base de aceite, básicamente en razón de tener un punto de ebullición más alto que aquellas ocasionadas por escaldaduras con agua u otros líquidos. Son igualmente consideradas quemaduras térmicas aquellas ocurridas con objetos sólidos calientes como metal sólido, plástico caliente, vidrio o piedra.

\section{Quemaduras químicas.}

Son causadas por ácidos fuertes, álcalis y compuestos orgánicos, comúnmente hallados en productos de limpieza para el hogar (para eliminar el óxido, para limpieza del baño) que producen la coagulación de proteínas, que resulta en lesiones menos extensas, y, a menos que se neutralicen, destruyen el tejido y continúan haciendo daño hasta 72 horas. Las quemaduras químicas más profundas, causadas por álcalis, como los que contienen los limpiadores de hornos y fertilizantes, por ejemplo, producen necrosis del tejido por licuefacción, haciendo que dicha sustancia penetre más profundamente en los tejidos. También figuran los compuestos orgánicos (en los que se incluye la gasolina y desinfectantes químicos), que pueden ocasionar necrosis de coagulación severa y generar escaras, entendidas como aquella capa de tejido grueso y no viable, que normalmente está presente en quemaduras de espesor total. 


\section{Limpiezas quirúrgicas en pacientes quemados}

Vol. 3, núm. 3., (2019)

Yessenia Isabel Sarchi Guayasamin; Olmedo Xavier Ruiz Lara; Paulina Elizabeth Durán Mora;

Jessica Karina Herrera Jaramillo

\section{Quemaduras eléctricas.}

Ocurren en alta tensión (1,000 voltios o más) o baja tensión (menos de 1,000 voltios). Básicamente, éste tipo de lesiones, al producir fibrilación ventricular o parálisis de los músculos respiratorios, pueden ocasionar la muerte. Aunque las arritmias se observan con mayor frecuencia en lesiones de alto voltaje, pueden igualmente propias en bajo voltaje.

El alcance del daño causado por una quemadura eléctrica puede parecer inicialmente menor: el paciente solo puede tener pequeñas heridas de entrada y salida. El daño extenso puede aparecer dentro de varios días o semanas, un fenómeno conocido como el efecto iceberg porque la superficie de la piel muestra poca lesión y oculta una lesión masiva debajo. En lugar de conducir la electricidad, los huesos, los músculos, los tendones y las grasas responden a las lesiones eléctricas produciendo calor. La mayoría de las lesiones ocurren en los músculos que rodean los huesos largos. (pág. 17)

Quemaduras por radiación.

Son el resultado de la exposición a la luz solar, cabinas de bronceado, rayos X o emisiones o explosiones nucleares. La radiación ionizante puede producir daño tisular directamente al golpear una molécula vital como el ADN. La radioterapia puede causar quemaduras de espesor total.

Quemaduras por humo e inhalación. 


\section{Limpiezas quirúrgicas en pacientes quemados}

Vol. 3, núm. 3., (2019)

Yessenia Isabel Sarchi Guayasamin; Olmedo Xavier Ruiz Lara; Paulina Elizabeth Durán Mora; Jessica Karina Herrera Jaramillo

Es posible que ocurra a la par de una térmicas o químicas. Los signos de quemaduras por inhalación son quemaduras faciales, ronquera, hollín en la nariz o la boca, carbón en el esputo, edema labial y cejas chamuscadas o vello nasal, y ello es si la persona presenta signos de quemadura térmica. Otro ejemplo pudiera ser la quemadura por inhalación asociada a la fabricación de metanfetamina ilegal, que igualmente puede estar vinculada a quemaduras térmicas y químicas. Todo paciente lesionado de quemadura por inhalación requerirá necesariamente de inmediatas intervenciones respiratorias, tales como: broncoscopia, intubación endotraqueal y medición de los niveles de carboxihemoglobina (COHgb).

\section{Quemaduras por congelación.}

Debido a que la congelación causa daños en la piel, algunos pacientes son tratados en la UCI como pacientes con quemaduras, aunque el tratamiento inicial para la congelación es diferente al de las quemaduras. (pág. 18)

Es el resultado que se obtiene en cualquier área del cuerpo al exponerlas a temperaturas muy frías, puesto que el flujo de sangre a la capa externa de la piel se reduce y el tejido de la piel, congelado o no, comienza a morir, pudiendo ocasionar un daño tisular temporal o permanente. Las áreas comúnmente más afectadas son los dedos de las manos y los pies, la barbilla, los lóbulos de las orejas, las mejillas y la nariz. La congelación puede progresar a necrosis, gangrena, hipotermia y paro cardíaco si no es tratada.

\section{Grado del Daño}




\section{Limpiezas quirúrgicas en pacientes quemados}

Vol. 3, núm. 3., (2019)

Yessenia Isabel Sarchi Guayasamin; Olmedo Xavier Ruiz Lara; Paulina Elizabeth Durán Mora; Jessica Karina Herrera Jaramillo

Básicamente, las quemaduras pueden comprenderse dentro de dos grupos que son: las menores y mayores. Las de daño menor comprenden las quemaduras de primer grado en cualquier parte del cuerpo y las quemaduras de segundo grado de menos de 2 a 3 pulgadas ( 5 a 7 centímetros) de ancho; mientras que las de mayor daño incluyen, las quemaduras de tercer grado; las de segundo grado de más de 2 a 3 pulgadas (5 a 7 centímetros) de ancho o de éste mismo grado en el área de las manos, pies, rostro, ingle, glúteos o sobre una articulación importante. (Medline Plus, 2018)

Quemadura de primer grado. Daña solo la capa externa de la piel (epidermis), pudiendo ocasionar enrojecimiento y dolor.

Quemadura de segundo grado. Afecta dos capas de la piel, tanto la epidermis como la dermis. Conducente de hinchazón y hacer que la piel se vea roja, blanca o manchada, así mismo, potencialmente se originan ampollas e intenso dolor. También es posible que las profundas quemaduras de este tipo dejen cicatrices.

Quemadura de tercer grado. Esta llega hasta la capa adiposa que se ubica debajo de la piel. Los segmentos quemados pueden quedar de color negro, marrón o blanco. Igualmente, en estos casos, posiblemente se perciba con una apariencia coriácea de la piel. Este tipo de quemaduras alcanzan la destrucción de los nervios razón por la que se puede generar un característico entumecimiento. (Mayo Clinic, 2019)

Tabla 1. Clasificación del paciente quemado

\begin{tabular}{|l|l|l|}
\hline Quemado Menor & Quemado Moderado & \multicolumn{1}{c|}{ Quemado Mayor } \\
\hline $\begin{array}{l}\leq 15 \% \text { Superficie corporal quemada } \\
\text { de primer o segundo grado en } \\
\text { adulto, }\end{array}$ & $\begin{array}{l}\text { De } 15 \text { a } 25 \% \text { Superficie corporal } \\
\text { quemada de segundo grado en } \\
\text { adulto, }\end{array}$ & $\begin{array}{l}>25 \% \text { Superficie corporal quemada } \\
\text { de segundo grado en adulto. }\end{array}$ \\
$>20 \%$ Superficie corporal quemada \\
\hline
\end{tabular}




\section{Limpiezas quirúrgicas en pacientes quemados}

Vol. 3, núm. 3., (2019)

Yessenia Isabel Sarchi Guayasamin; Olmedo Xavier Ruiz Lara; Paulina Elizabeth Durán Mora; Jessica Karina Herrera Jaramillo

\begin{tabular}{|c|c|c|}
\hline $\begin{array}{l}\leq 10 \% \text { Superficie corporal quemada } \\
\text { de primer o segundo grado en niño, } \\
\leq 2 \% \text { Superficie corporal quemada } \\
\text { de tercer grado en niño o Adulto } \\
\text { que no } \\
\text { incluya ojos, orejas, cara o } \\
\text { genitales, }\end{array}$ & $\begin{array}{l}\text { De } 10 \text { a } 20 \% \text { Superficie corporal } \\
\text { quemada de segundo grado en niño. } \\
\text { De } 2 \text { a } 10 \% \text { Superficie corporal } \\
\text { quemada de tercer grado en niño o } \\
\text { adulto } \\
\text { que no incluya ojos, orejas, cara o } \\
\text { genitales }\end{array}$ & $\begin{array}{l}\text { de segundo grado en niño. } \\
>10 \% \text { Superficie corporal quemada } \\
\text { de tercer grado en adulto o niño. } \\
\text { Cualquier quemadura que incluya } \\
\text { ojos, orejas, cara, genitales, manos } \\
\text { o pies. } \\
\text { Trastornos por inhalación. } \\
\text { Quemaduras eléctricas. } \\
\text { TCE o traumas mayores que } \\
\text { incluyan lesiones por quemaduras } \\
\text { complicadas. } \\
\text { Pacientes de riesgo por } \\
\text { complicaciones debidas a } \\
\text { patologías medicas asociadas: } \\
\text { Diabetes, EPOC, ACV, procesos } \\
\text { neoplásicos, alteraciones } \\
\text { psiquiátricas, entre otras. }\end{array}$ \\
\hline
\end{tabular}

Nota: adaptado de (Muñóz, 2011)

Ahora bien, teniendo una idea clara de lo que básicamente significa una quemadura y de sus tipos y grados de daños en los diversos tejidos, podemos entrar en la materia que nos ocupa, la limpieza quirúrgica en pacientes quemados.

Ante todo, debe tenerse presente que el manejo quirúrgico de las quemaduras se asume fundamentalmente como un mecanismo preventivo contra la infección, así lo dan a entender Ramírez, Rivera, Lorenzo, \& Uribe (1997) quienes afirman que "El desbridamiento y escisión temprana de la escara y la colocación de injertos es lo más efectivo para disminuir las posibilidades de sepsis en el paciente quemado." (p. 45)

De acuerdo con la acepción de Santamaría (s.f.) la limpieza quirúrgica de tejidos blandos, se trata de la extirpación de todo aquel tejido que, bajo una anestesia local, regional o general 


\section{Limpiezas quirúrgicas en pacientes quemados}

Vol. 3, núm. 3., (2019)

Yessenia Isabel Sarchi Guayasamin; Olmedo Xavier Ruiz Lara; Paulina Elizabeth Durán Mora; Jessica Karina Herrera Jaramillo

(dependiendo de su ubicación y características), se encuentre desvitalizado, a fines de disminuir la sintomatología dolorosa o compresiva asociada al traumatismo en particular, y favoreciendo una mejor cicatrización. No obstante, aclara el especialista que, entre las posibles consecuencias que se originan de este proceso, destaca la ineludible molestia en las zonas intervenidas (propias de la técnica aplicada o por la adaptación de los músculos del mismo sitio) que pueden prolongarse por semanas, meses e incluso perenes.

En base a lo antes expuesto, bien se puede deducir que la limpieza quirúrgica de un tejido afectado por una quemada tiene como objetivo primordial la obtención de una cicatrización óptima, en razón de la supresión quirúrgica tanto de cuerpos extraños como de restos necróticos, lo que induce la aceleración de la reparación tisular y disminuye el riesgo de infección.

\section{Limpieza de las quemaduras}

De acuerdo a la idea de Berrocal, Guette, Rodríguez, Ruiz, \& Salgado (2011), respecto al manejo local de la quemadura, afirman que:

La limpieza de las áreas quemadas se realiza preferiblemente bajo sedación general, con irrigación abundante de suero fisiológico frío. Si es necesario utilizar jabón neutro para retirar elementos extraños. No está indicado frotar las zonas quemadas con apósitos o compresas secas, ya que ello aumenta el trauma tisular. Está contraindicado romper as vesículas y resecarlas en la palma de las manos o planta de los pies. Después de realizada la limpieza, el paciente debe ser colocado sobre ropa estéril. Decidir si es pertinente una curación con método oclusivo o método expuesto, utilizando medicamentos tópicos. El método oclusivo se recomienda en todas las áreas 


\section{Limpiezas quirúrgicas en pacientes quemados}

Vol. 3, núm. 3., (2019)

Yessenia Isabel Sarchi Guayasamin; Olmedo Xavier Ruiz Lara; Paulina Elizabeth Durán Mora; Jessica Karina Herrera Jaramillo

de articulaciones a fin de mantener la extensión de las extremidades. El método de curación expuesta se recomienda en cara y genitales. (p. 323)

Baltà \& Valls (2018) indican que, en quemaduras limpias se procede con una limpieza por arrastre mediante irrigación con SSF (solución salina fisiológica). En caso contrario, o porque se ubiquen en zonas como las de los pies, manos, genitales, zona perianal y axilas (consideradas de riesgo), la limpieza se efectúa con jabón indicado y un antiséptico tipo clorhexidina, solución de polihexanida decilenamidopropilbetaína, povidona yodada o de similar espectro antibacteriano.

\section{Limpieza Quirúrgica}

Son técnicas propias en quemaduras de segundo grado superficial / profundo y tercer grado.

Al respecto, el Servicio Andaluz de Salud de España (2011) mediante su guía práctica recomienda: se irrigue con agua a temperatura ambiente, limpie la piel con agua del grifo, agua hervida, agua destilada o suero salino, no aplique agua muy fría o helada, haga el lavado/ aseo por ducha / irrigación, use clorhexidina como antiséptico de elección en las primeras fases de curación, seque la piel sin frotar la zona lesionada, retire el vello que rodea la quemadura, elimine el vello cortándolo con tijeras en vez de rasurar, retire apósitos, gasas y vendajes por capas, utilice la mínima fuerza mecánica para la limpieza, secado y retirada de los apósitos, retire ampollas o $\neg$ flictenas. (p. 45 - 48)

En otro destacado material titulado "Guía Clínica. Manejo del paciente gran quemado (2da. edición)" del Ministerio de Salud de Chile (2016) se encuentra una matiz más detallada que aclara el tratamiento quirúrgico de este tipo de afecciones, al respecto indicandose que la reparación de 


\section{Limpiezas quirúrgicas en pacientes quemados}

Vol. 3, núm. 3., (2019)

Yessenia Isabel Sarchi Guayasamin; Olmedo Xavier Ruiz Lara; Paulina Elizabeth Durán Mora;

Jessica Karina Herrera Jaramillo

la cubierta cutánea en quemaduras puede conseguirse de manera espontánea o bien puede necesitar de procedimientos quirúrgicos. El potencial regenerativo de la piel, que a su vez depende de la población celular indemne (principalmente de queratinocitos) y de su viabilidad, estando ésta fundamentalmente condicionada por la eficacia de la perfusión, es lo que se concibe como el factor determinante del pronóstico de una quemada. Por lo tanto, dependiendo de la profundidad de ésta es que entonces se dirigirán los procedimientos reparativos.

Allí mismo, se recomienda hacer protección de los queratinocitos viables, en las quemaduras de espesor parcial, en las que la población de queratinocitos es determinada como susceptible de repararse espontáneamente (A - ABA). Mientras que la escarectomía precoz y cubierta inmediata es recomendada en las quemaduras de espesor total o en aquellas de espesor parcial en las que la población de queratinocitos indemnes se considere insuficiente para conseguir la epidermización espontánea o no hay condiciones para asegurar su perfusión (B - ABB).

De la misma manera se extrae que, inicialmente, toda limpieza quirúrgica debe hacerse en el paciente previamente estabilizado a nivel hemodinámico, habiendo eliminado el dolor y con evacuación vesical funcionante, solamente exceptuando aquellos casos en que las lesiones locales requieran tratamiento de urgencia y sean condicionantes de la inestabilidad.

La preparación inicial sobre campos estériles impermeables incluye las siguientes actividades: aseo que elimine suciedad, restos de ropas u otros materiales extraños; aseo por arrastre con agua o solución fisiológica; rasurado cuidadoso de zonas quemadas y su contorno (Incluir cuero cabelludo si está comprometido; secado y preparación de campo estéril definitivo. (p. 33) 


\section{Limpiezas quirúrgicas en pacientes quemados}

Vol. 3, núm. 3., (2019)

Yessenia Isabel Sarchi Guayasamin; Olmedo Xavier Ruiz Lara; Paulina Elizabeth Durán Mora; Jessica Karina Herrera Jaramillo

En esta quía se deja claro que "el aseo quirúrgico permite establecer el diagnóstico de extensión, profundidad, compresión, restricción y el pronóstico inicial" considerándose oficios mínimos, para el aseguramiento de la estabilidad y supervivencia del paciente, tales como:

- Retiro de tejido desvitalizado, flictenas y otros contaminantes.

- Lavado con suero fisiológico abundante.

- Escarotomía o fasciotomía, desbridamiento de compartimentos musculares.

- Cuando está indicado.

No corresponde realizar en esta etapa escarectomía, amputaciones ni otras cirugías de la especialidad, ni de mayor envergadura, salvo que fueren necesarias para la sobrevida del paciente. (Ministerio de Salud de Chile, 2016)

\section{Desbridamiento}

"El desbridamiento es la eliminación de tejido infectado, dañado o muerto para que una herida pueda sanar correctamente. Es posible que usted necesite más de un desbridamiento". (Drugs, 2019)

En la misma fuente bibliográfica se encuentra que, existen varios métodos selectivos de desbridamiento, que puedes ser: 


\section{Limpiezas quirúrgicas en pacientes quemados}

Vol. 3, núm. 3., (2019)

Yessenia Isabel Sarchi Guayasamin; Olmedo Xavier Ruiz Lara; Paulina Elizabeth Durán Mora; Jessica Karina Herrera Jaramillo

- El autolítico, usa los propios líquidos de la herida para separar el tejido sano del muerto. Su herida se cubre con vendajes para evitar que la herida se humedezca. Las proteínas del líquido de su herida transformarán el tejido muerto y duro en líquido. Después le lavan su herida para eliminar los residuos de tejido licuado.

- El de biocirugía, usa larvas de gusano estériles, que liberan enzimas que degradan el tejido muerto. Las larvas digieren bacterias en la herida y las enzimas evitan que se desarrollen nuevas bacterias en la herida.

- El enzimático, usa medicamentos de proteínas de enzimas que funcionan digiriendo y fundiendo el tejido.

- El hipobárico, usa un vendaje con espuma y un tubo de succión en la herida limpia. Se sella con una venda transparente que se puede ver a través de esta para crear succión en la herida. La succión saca y elimina los tejidos muertos y los líquidos adicionales. Esto provoca el crecimiento de tejido nuevo y saludable.

- El quirúrgico, se usa para la heridas grandes o severamente infectadas. Se usan instrumentos quirúrgicos, como un escalpelo, pinzas y tijeras.

\section{Las flictenas y epitelio necrosado restante}

Este particular aspecto se ha considerado en la obra de Baltà \& Valls (2018), puesto que indican que todas las flictenas (flictenectomía) deben desbridarse y todo el tejido orgánico desvitalizado ha de ser retirado; agregando que, aún y cuando es insuficiente la evidencia científica que afiance la flictenectomía, es indiscutible el acuerdo entre otros expertos al lograr identificar que dicha práctica aporta mayores beneficios. Sin embrago, aclaran que, respecto a los restos de 


\section{Limpiezas quirúrgicas en pacientes quemados}

Vol. 3, núm. 3., (2019)

Yessenia Isabel Sarchi Guayasamin; Olmedo Xavier Ruiz Lara; Paulina Elizabeth Durán Mora; Jessica Karina Herrera Jaramillo

epidermis desvitalizada que quedan fuertemente adheridas a la dermis subyacente "se irán desprendiendo paulatinamente de forma incruenta en las posteriores curas."

Del mismo aporte se logra identificar entender varias desventajas que surgen al conservar la flictena sin desbridar, siendo estas las siguientes:

- Se dificulta la disminución de la presión en los tejidos dañados y, por ende, continúa agudizándose la lesión, pudiendo entonces convertirse en una lesión de segundo grado profundo, cuando inicialmente era de segundo grado superficial.

- Favorece que pueda desarrollarse una infección autóloga (a partir de la flora saprófita), tampoco protege de una infección cruzada.

- El líquido de las flictenas que se genera por la destrucción celular colindante, contiene sustancias citotóxicas que, en base a evidencias, se puede asumir como un factor de necrosis tumoral, y de igual forma pudiera esto ser considerado como la causa de la profundización de la lesión.

- Impide que el tratamiento tópico antibacteriano pueda llegar al lecho de la herida.

\section{Escisión Quirúrgica}

La escisión quirúrgica "es la extirpación de tejido utilizando un cuchillo afilado (bisturí) u otro instrumento cortante". (Enciclopedia Médica ADAM, 2017)

En paciente quemados, con lesiones de gran extensión y profundidades mayores a tercer grado, en alguna medida requieren de algún tipo de manejo quirúrgico. Una de las técnicas que se realizan para la remoción de tejido muerto para la aplicación de otros tratamientos, es la escisión. 


\section{Limpiezas quirúrgicas en pacientes quemados}

Vol. 3, núm. 3., (2019)

Yessenia Isabel Sarchi Guayasamin; Olmedo Xavier Ruiz Lara; Paulina Elizabeth Durán Mora; Jessica Karina Herrera Jaramillo

Hay múltiples formas de escisión para pacientes quemados, para determinar la adecuada es fundamental la valoración de la profundidad de las quemaduras. Entre las principales encontramos:

\section{Escisión Fascial.}

Vélez-Palafox (2014) mencionan en su trabajo que esta técnica decide escindir directamente hasta fascia, respetándola, y donde se reseca la piel y el tejido celular subcutáneo. "Está indicada en pacientes con quemaduras muy extensas que ponen en peligro su vida. Esta disminuye el sangrado ya que únicamente se exponen los vasos perforantes los cuales son de mayor calibre y se realiza hemostasia más fácilmente”. (p. S-227)

\section{Escisión Tangencial.}

En esta otra técnica "se reseca con dermatomos manuales por capas el tejido quemado y necrótico hasta localizar tejido viable. Una de las ventajas de esta técnica es que respeta parte del tejido celular subcutáneo sin llegar la mayoría de las veces a fascia”. Este procedimiento expone un plano con tejido viable, que se encuentra listo para ser injertado, además preserva un mejor contorno corporal. (Vélez-Palafox, 2014, p. S-227)

\section{Conclusión}

La limpieza quirúrgica permite preparar el área para realizar injertos (en los casos en que el paciente los requiera) ya que en quemaduras de mayor gravedad aumenta las posibilidades de supervivencia, disminuye la estancia intrahospitalaria y mejora los resultados tanto funcionales 


\section{Limpiezas quirúrgicas en pacientes quemados}

Vol. 3, núm. 3., (2019)

Yessenia Isabel Sarchi Guayasamin; Olmedo Xavier Ruiz Lara; Paulina Elizabeth Durán Mora; Jessica Karina Herrera Jaramillo

como de rehabilitación del paciente quemado, por ende, proveen una mejor integración a su vida cotidiana.

En tal sentido, este procedimiento vendría entonces a representar un papel fundamental en el manejo de las quemaduras, y es uno de los más importantes en la medicina actual.

Un adecuado tratamiento quirúrgico garantiza resultados eficientes y reduce los riesgos de complicaciones, tales como la pérdida de la función de un miembro o parte del cuerpo y en el peor de los casos la muerte.

\section{Referencias bibliográficas}

Córdova, C., Moreno, Á., Maruri, P., \& Criollo, M. (Julio de 2019). La bioimpresora al servicio de los pacientes con quemaduras. Reciamuc, 3(3), 107-121. Recuperado el 02 de 10 de 2019, de http://www.reciamuc.com/index.php/RECIAMUC/article/view/269/285

Enciclopedia Médica ADAM. (09 de Septiembre de 2017). MedlinePlus. Recuperado el 09 de Octubre de 2019, de https://medlineplus.gov/spanish/ency/article/002305.htm

Enciclopedia Médica ADAM. (05 de Diciembre de 2018). MedlinePlus. (MedlinePlus, Ed.) Obtenido de https://medlineplus.gov/spanish/ency/patientinstructions/000739.htm

Enciclopedia Médica ADAM. (04 de Abril de 2019). MedlinePlus. (MedlinePlus, Editor, \& B. N. Unidos, Productor) Recuperado el 05 de Octubre de 2019, de https://medlineplus.gov/spanish/burns.html

Enciclopedia Médica ADAM. (20 de Agosto de 2019). MedlinePlus. (MedlinePlus, Editor, \& B. N. Unidos, Productor) Recuperado el 07 de Octubre de 2019, de https://medlineplus.gov/spanish/pruebas-de-laboratorio/evaluacion-de-quemaduras/

Mayo Clinic. (15 de Junio de 2019). Mayo Clinic. Recuperado el 01 de 10 de 2019, de mayoclinic.org/es-es: https://www.mayoclinic.org/es-es/diseasesconditions/burns/symptoms-causes/syc-20370539 


\section{Limpiezas quirúrgicas en pacientes quemados}

Vol. 3, núm. 3., (2019)

Yessenia Isabel Sarchi Guayasamin; Olmedo Xavier Ruiz Lara; Paulina Elizabeth Durán Mora; Jessica Karina Herrera Jaramillo

Medline Plus. (24 de Mayo de 2018). Medline Plus, Enciclopedia Médica. Recuperado el 01 de 10 de 2019, de https://medlineplus.gov/spanish: https://medlineplus.gov/spanish/ency/article/000030.htm

Organización Mundial de la Salud. (2007). Organización Mundial de la Salud. Recuperado el 09 de Octubre de 2019, de https://www.who.int/hac/techguidance/Disasters_management_guidelines_ESP.pdf

Organización Mundial de la Salud. (06 de Marzo de 2018). Organización Mundial de la Salud. Recuperado el 01 de Octubre de 2019, de https://www.who.int/es/news-room/factsheets/detail/burns

Ramírez, C., Rivera, J., Lorenzo, M. C., \& Uribe, J. (1997). Manejo de Quemados. En C. Ramírez, J. Rivera, M. C. Lorenzo, \& J. Uribe, Guías de Práctica Clínica basadas en la Evidencia (Profecto ISS. ASCOFAME) (Instituto de Seguros Sociales - Asociación Colombiana de Facultades de Medicina (ASCOFAME) ed., págs. 1-82). Bogotá, Colombia: Maldonado S.A. $\begin{array}{lllllll}\text { Recuperado el } & 02 & \text { de } & 10 & \text { de } & 2019, & \text { de }\end{array}$ http://ascofame.org.co/biblioteca/Documentos/53.\%20MANEJO\%20DE\%20QUEMADOS .pdf

Santamaría, L. (s.f.). Instituto de Ortopedia y Traumatología (IOT). Recuperado el 01 de 10 de 2019, de traumatologia.com.ec: http://traumatologia.com.ec/procedimientos/

Servicio Andaluz de Salud de España. (2011). portal.guiasalud.es. (D. G. Salud, Ed.) Recuperado el 02 de Octubre de 2019, de https://portal.guiasalud.es/wpcontent/uploads/2019/01/GPC_485_Quemados_Junta_Andalucia_completa.pdf

Vélez-Palafox, M. (2014). Tratamiento quirúrgico de las quemaduras y protocolo de salvamento. Revista Mexicana de Anestesiología, 37(1), S226-S229. Recuperado el 09 de Octubre de 2019, de https://www.medigraphic.com/pdfs/rma/cma-2014/cmas141bh.pdf 